\title{
Quaderni
}

QUADERNI Communication, technologies, pouvoir

89 | Hiver 2015-2016

Penser l'avenir : le CESTA, un think tank atypique

\section{"Organisation, anti-organisation" : un changement de paradigme pour penser l'innovation et la prospective}

\section{Franck Cormerais}

\section{(2) OpenEdition \\ Journals}

Édition électronique

URL : http://journals.openedition.org/quaderni/954

DOI : 10.4000 /quaderni.954

ISSN : 2105-2956

Éditeur

Les éditions de la Maison des sciences de l'Homme

\section{Édition imprimée}

Date de publication : 5 janvier 2016

Pagination : 43-54

Référence électronique

Franck Cormerais, «"Organisation, anti-organisation" : un changement de paradigme pour penser l'innovation et la prospective », Quaderni [En ligne], 89 | Hiver 2015-2016, mis en ligne le 05 janvier 2018, consulté le 19 avril 2019. URL : http://journals.openedition.org/quaderni/954 ; DOI : 10.4000/ quaderni.954 


\section{$D$ ossier}

\section{"Organisation,}

anti-organisation" : un changement de paradigme pour penser l'innovation et la prospective

\section{Franck \\ Cormerais}

\author{
Professeur des Universités \\ Bordeaux-Montaigne \\ Laboratoire MICA
}

La question de l'organisation se repose en ce début du XXI ${ }^{e}$ siècle pour des raisons diverses. La mondialisation actuelle suscite une reconfiguration des territoires politiques, géographiques, économiques, informationnels, technologiques. Les réseaux appellent des ordres de grandeur et des processus de valorisation nouveaux. Partant d'une relecture d'un ouvrage (Stourdzé, 1973), nous soulignerons à la fois l'apport des propositions contenues dans cet opus et la richesse des perspectives qu'il ouvre. En ce moment contemporain de désordre du monde, la position de Stourdzé peut-elle aider, face à l'autotranscendance des marchés, à penser autrement l'innovation et la prospective ? La mystification d'une croissance illimitée ainsi qu'un changement nécessaire de paradigme pour s'installer dans la durée soutenable rendent-ils, aujourd'hui encore, intéressants les partis pris théoriques de l'ouvrage?

Dans la première partie de cet article l'organisation apparaît comme la question qui introduit une approche anthropologique originale du pouvoir. Dans la seconde partie nous verrons comment la mutation du système de production s'envisage comme une limitation du sacrifice. En conclusion, nous observerons en quoi la position de Stourdzé oscille entre une critique active et un pessimisme foncier. Cette ambivalence prépare une rupture qu'inscrira par la suite le CESTA. Fort d'une réflexion sur l'organisation, à nos yeux toujours d'actualité, Stourdzé se transformera en « capitaine de vaisseau » d'une structure qui formulera une réponse concrète aux conclusions d'un ouvrage qui signait la fin d'une période politique, où la contestation radicale était de mise. Stourdzé entrera finalement à la fin des années 70 dans 
une nouvelle phase de son parcours : il devient lui-même organisateur et pratiquera autrement l'innovation et la prospective.

\section{L'organisation comme la question centrale}

Dans cette partie nous montrerons comment la vision théorique de Stourdzé peut être interprétée à partir d'une audace conceptuelle qui renouvelle les perspective de l'approche organisationnelle. Cette perspective repose d'abord sur une critique du sociologisme. Elle souligne après Saint-Simon l'importance des organisations pour aborder la réforme de la société dans son ensemble. La question de l'organisation s'inscrit à la fois dans une filiation et un moment historique où l'industrialisation se transforme. L'organisation au tournant des années 70 redevient une question centrale d'un courant de la pensée critique. Organisation, anti-organisation est publiée en 1973, année de la publication de La convivialité d'Illich et de Small is beautiful de Schumacher. La position de Stourdzé s'inscrit dans la perspective d'une « troisième voie » (Sik, 1974) qui pose une alternative au marxisme et au libéralisme. Une critique de l'organisation se comprend, in fine, comme la recherche de nouveaux espaces de liberté.

\section{L'organisation réinterprétée par l'information}

Stourdzé aborde les organisations d'une manière élargie: elles canalisent, endiguent et contrôlent les forces sociales ; elles codifient, quadrillent et centralisent. Les organisations sont par la variété de leurs fonctions des institutions qui se comprennent comme le milieu de l'adaptation des fins aux moyens. Pour cela, elles produisent un imaginaire qui capte le désir. En leur cœur se produit un paradoxe : «l'organisation tire sa force de ce processus double de destruction et d'unification. D'un côté les membres sont brisés, mais de l'autre l'imaginaire organisationnel leur garantie l'unité corporelle» (Stourdzé, 1973,14). Issue d'une longue histoire qui résulte d'un processus d'intégration, les organisations reposent sur une synthèse du militaire et du religieux. Leurs dynamiques produisent des coupures et des limites car elles séparent et découpent; elles rythment les échanges et les distributions avec l'extérieur. L'organisation structure un réseau nécessitant un besoin d'énergie pour alimenter le fonctionnement de sa clôture protectrice. Face à l'entropie de toute structure, le besoin extérieur alimente l'ordre. Pour s'éloigner des modèles historiques, Stourdzé reprend l'information pour aborder les processus d'intégration et de désintégration. Comment s'élabore progressivement sa position ? Stourdzé signale d'entrée sa dette à un courant de pensée originale, « l'analyse institutionnelle » (Stourdzé, 1973,8), qui, complétée par la perspective informationnelle, débouche sur une orientation analytique renouvelée. Dans la composition de son ouvrage, la question organisationnelle se place au croisement de trois strates. L'organisation est ainsi posée dans un horizon complexe, à la fois matériel, informationnel et symbolique (cf. tableau 1).

Au travers de trois strates s'opère un déblocage $\mathrm{du}$ « sociologisme » par une approche informationnelle, où les notions d'ordre et de désordre deviennent des clés pour aborder les deux logiques de l'organisation: agrégation et séparation. Autour des opérations de capture et d'extraction, l'organisation se trouve reliée à une énergie nécessaire à toute mise en production. L'investis- 


\begin{tabular}{|l|l|l|l|}
\hline Strates & Champ & Manifestation & Sortie \\
\hline Matériel & Physique, bio et technique & Énergie et action & Distribution \\
\hline Informationnel & $\begin{array}{l}\text { Information de structure et } \\
\text { information de contenu }\end{array}$ & Codification et Circulation & Loi, norme \\
\hline Symbolique & Psychique & Virtualisation et pensée & $\begin{array}{l}\text { Autorité, pouvoir } \\
\text { contrôle }\end{array}$ \\
\hline
\end{tabular}

Tableau $n^{\circ} 1$ : Les trois strates de l'organisation

sement organisationnel délivre un premier niveau de lecture psychosociologique, où la canalisation de l'énergie psychique exige une soumission à un réseau du pouvoir et un centre de coordination. Ce réseau de répartition s'entend comme une structure de la relation au pouvoir que l'on peut nommer « technologico-politique ».

Le technologico-politique inscrit la dimension technique dans le mode opératoire du pouvoir, indissociable des tâches d'extraction et de branchement sur l'extérieur. Les principes de l'organisation, issus de la théorie de l'information qui inspire le titre de l'ouvrage, permettent de s'éloigner de la dialectique marxiste de la lutte des classes qui primait dans les années 70. L'ordre et le désordre informationnel viennent ainsi préciser les échanges régulés (codification, distribution et mesure, contrôle) entre les strates matérielles et symboliques. L'existence de conflits et de tensions ne disparaît pas pour autant, mais se trouve réinterprétée d'une autre manière.

\section{Le sacrifice comme approche originale de l'organisation}

L'approche informationnelle de l'organisation va permettre ensuite à Stourdzé de formuler une hypothèse plus globale : le désordre et l'ordre vont devenir des marqueurs essentiels de l'activité économique. La référence classique à la théorie de l'équilibre général cesse d'être centrale alors pour laisser place à une lecture de la « dépense » qui guidera l'analyse. L'économie fonctionne désormais sur la relation dépense/ investissement dans la perspective d'une économie générale, après Georges Bataille (Bataille, 1949).

À l'aide de la théorie de l'information et de la perspective ouverte par la Part Maudite, Stourdzé franchit un pas. Ce mouvement s'opère dans une approche originale des organisations: le sacrifice devient une pierre angulaire qui éclaire la dépense. Il s'agit de replacer l'approche informationnelle dans le cadre de la société. L'organisation ne saurait se réduire à un modèle cybernétique. La relation de l'homme à la société doit être complétée par une anthropologie qui ne peut ignorer le sacrifice analysé par Marcel Mauss (Mauss, 1968). L'analyse du sacrifice et de la violence trouve sa place dans l'examen du champ organisationnel où « les miroitements glacés de la rationalité ne peuvent masquer bien longtemps les dispositifs violents et sacrificiels. » (Stourdzé, 1973, 14). 
Cette position renforce une critique du capitalisme par la présentation des phénomènes de transfert et l'étude des dispositifs de contrôle des normes : capitaliser c'est avant tout organiser un report de la jouissance par où s'inscrit son sacrifice. Le don, la dépense et l'investissement relient le symbolique au matériel. Penser l'organisation implique une logique sacrificielle qui revient sur la question de l'origine de la violence. Cette ouverture anthropologique s'inscrit dans un écart au marxisme ; le sacrifice est alors posé dans sa double relation au travail et à l'économie non plus seulement dans l'horizon des rites ou des religions. Le travail est à la fois le sacrifice du repos, de la liberté (pour le travailleur) et le sacrifice de l'abstinence (pour le capitaliste) qui thésaurise afin de pouvoir accumuler. Ainsi, «Pour Marx, le travail, le lieu de travail n'a de sens que comme instance de transformation de la force de travail (énergie); Marx ignore le travail comme organisation imaginaire, comme lieu justement de sacrifice, de l'investissement, du désir, de la dilapidation de l'énergie (de la mort). À la limite, le travail comme énergie peut être fourni par d'autres sources que les travailleurs [...]. » (Stourdzé, 1973, 42). Cette critique du marxisme développe une conception autre de l'entreprise, où "l'organisation est moins le lieu de la production de la marchandise que le lieu de l'asservissement, le lieu du sacrifice. » (Stourdzé, 1973, 43).

Stourdzé constate de surcroît que les forces productives deviennent de moins en moins importantes à mesure que la productivité liée aux machines se développe ; ce qui laisse du temps « hors circuit » (Stourdzé, 1973, 44) de la valeur économique. Nous trouvons là un passage qui met en crise les limites de la valeur travail apprécié comme un quantum. L'importance de l'imaginaire et du désir se trouve soulignée comme nécessité qui renvoie à la dimension symbolique de l'organisation. Ainsi, ce qui échappe au circuit de la valeur économique du travail devient un enjeu décisif. L'organisation se comprend comme le lieu de la médiation, elle structure les relations entre les corps et le monde des signes; elle distribue l'information entre le matériel et le symbolique. Le paradoxe propre au sacrifice est de présenter une destruction masquée par la création de marchandise. Finalement, la double destruction envisagée du point de vue du travail et de celui du capital présente le centre de l'organisation comme un lieu à partir duquel s'organise le sacrifice. Stourdzé pense alors l'aliénation de son époque comme capitalisme de la "séduction » (Stourdzé, 1973,116) qui fonctionne en captant le désir ; il devance ainsi les analyses de Baudrillard (1980) et de Clouscard (1981).

\section{La mutation de la production comme limi- tation possible du sacrifice}

Dans la seconde partie de l'ouvrage, en poursuivant son analyse du capitalisme, Stourdzé apporte une réponse à la dissolution des rapports fondamentaux du salariat que dévoile la montée progressive de l'automatisation. Il souligne l'importance des gains de productivité que permet le passage de l'électricité à l'électronique puis à l'informatique. Sa vision de la technologie invalide la théorie dite du " déversement » (Sauvy, 1980) dans laquelle l'emploi perdu dans une filière va rejoindre de nouvelles activités. Nous allons exposer les conséquences du principe sacrificiel à l'œuvre dans les organisations, 
maintenant compris comme opérateur d'une reformulation globale de la question organisationnelle.

\section{Le don des machines et l'élaboration de zones différentielles}

En réfléchissant à ce qu'apporte le machinisme, Stourdzé introduit un « don » des machines qui revient sur les mécanismes sacrificiels de l'échange. Il refuse le sacrifice compris comme «l'épargne de la jouissance au nom des objectifs planifiées ou des objets consommables » (Stourdzé, 1973,159). Contre les vues d'une prospective conduite par le plan, sa conception de l'organisation opère par coupures et limites; elle implique un rythme basé sur une dépense et un investissement qu'il convient d'envisager autour d'un autre modèle de la production et de l'échange, alternative au capitalisme consumériste des trente glorieuses. Entre ordre et désordre, il n'y a pas d'état stationnaire mais métastabilité. Entre input (entrée) et output (sortie) se développent des « zones différentielles » (Stourdzé, 1973, 24), où l'énergie se transforme dans un processus circulant entre intérieur et extérieur et structurant des moments d'expansion et de dépérissement.

Le concept de zone « différentielle » s'avère utile pour organiser les conversions et les transferts, les prélèvements et les captations des organisations. Ce concept autorise une relecture des principes de la décision et de la participation qui sont toujours liés à l'acceptation de produire au rythme demandé. En déplaçant les postulats de March et Simon dans une perspective qui s'apparente à celle de Sfez formulée à la même époque (Sfez, 1973), deux enseignements soulignent la démarche de
Stourdzé : d'abord un chemin est inscrit comme un possible grâce aux gains de productivité et, ensuite, la mutation de la production transforme la nature même du travail. Se référant aussi aux travaux de Pierre Naville (Naville, 1963) Stourdzé anticipe le rôle depuis lors grandissant de la robotique. L'enjeu de ces zones introduit un paradoxe qu'exprime un pharmacon par lequel la contrainte liée au travail peut aussi bien renforcer l'asservissement que le desserrer.

\section{L'importance du signe dans la coordination et le pouvoir}

Les « zones différentielles » se trouvent explicitées par le rôle grandissant de l'information. La dissolution du travail s'opère à travers une montée du traitement des signes mise en œuvre par les systèmes d'information. Le « quadrillage organisationnel » (Stourdzé, 1973, 55) produit de la continuité, en gommant tout risque d'interruption. Le contrôle de la distribution de l'information devient un leitmotiv dont s'empareront les sciences de la gestion, tandis que le statut de coordination est légué à l'ordinateur qui crée des isonomies entre les formes d'organisation. Cependant à l'inverse, l'ordinateur développe une rupture entre les membres de l'organisation cette fois. Ce n'est plus tant une logique traditionnelle de bureaucratisation qui prime, que la recherche de principes méta-organisationnels modifiant le rôle des chefs et des membres de la communauté de travail. Stourdzé souligne ainsi deux problèmes majeurs : «le fantasme pyramidal» qui assurerait une cohérence parfaite et, à l'inverse, l'illusion de la disparition des problèmes de coordination. C'est à travers la question de la coordination 
que se précise une critique de l'organisation hiérarchique. Coordonner devient le point-clé du pouvoir : "L'organisation rationnelle n'a nul besoin de centre et pourtant le centre est le pivot organisationnel, il faut comparer cette étrangeté au socialisme. Lui aussi se voulait organisation social ou le centre, l'État dépérit, et pourtant l'État y règne en maître. Le pouvoir s 'appréhende comme scandale. » (Stourdzé, 1973, 52).

Pour examiner les conditions de ce scandale, Stourdzé remarque que la détention d'information devient un vecteur de pouvoir. Mais, au-delà de ce constat, il lui semble que la pensée du pouvoir doit être renouvelée autour des notions de représentation, d'opinion, de demande. C'est donc une théorie de pouvoir en relation avec la communication qui se trouve ainsi esquissée. Cette théorie revient sur l'écoute et l'enregistrement des archives comme formes déterminantes d'assujettissement. Un changement dans les modes et outils de représentation implique un changement dans les actes et les conceptions du pouvoir. L'apparition de la géomatique en 1981 puis celle, plus récente, du data profiling confirment cette affirmation. De nouvelles cartes impliquent de nouvelles formes de gouvernementalité.

Sans entrer plus en avant dans l'exposition du pouvoir, remarquons deux points relatifs à la gestion de l'opinion dans les organisations. Le premier assemble des besoins et des volontés internes. Le second implique la coordination externe comme élément de justification. Ces traits précisent le «scandale »qu'évoque Stourdzé qui est lié à l'impossible liaison entre la coordination et la décentralisation car le
« centre est un lieu sacré » (Stourdzé, 1973, 83). Autour de l'assemblage et de la coordination se livre une voie entre le politique, l'éthique et le technologique. C'est dans cette direction qu'il faut aborder le technologico-politique pour échapper à un retour du théologico-politique.

\section{L'organisation entre économie et inflation sacrificielle}

L'ordre et le désordre puis l'approche sacrificielle de l'organisation autorisent, chez Stourdzé, sa tentative de repenser le centre des pouvoirs pour déboucher sur une régénération de la production à partir d'une réflexion qui relie innovation et création de valeurs. Stourdzé essaie de concevoir un moment organisationnel alternatif à la « destruction créatrice ». Le besoin de réinterpréter Schumpeter passe alors par une lecture de l'information qui rend souhaitable, sinon possible, une modification du sacrifice qui transforme la dégradation du temps.

« L'organisation induit la dégradation, usure de l'objet, obsolescence des techniques et des hommes. Elle n'échappe pas au deuxième principe de la thermodynamique, elle cherche à mettre en scène une rationalité inverse. La marchandise se veut toujours spectacle de prix, alors même que sa valeur décroît inéluctablement. Marx formulait cette logique en parlant de baisse tendancielle du taux de profit. Face à cette menace, l'organisation se crispe désespérément sur ce qui fonde sa légitimité: la régénération $d u$ temps. » (Stourdzé, 1973, 86).

En insistant sur l'aspect néguentropique d'une durée créatrice, les machines ouvrent la possi- 
bilité de nouveaux chemins de liberté par une autre organisation du temps. En réaffirmant la valeur d'usage dans sa durée face au mécanisme de l'usure nécessaire à l'investissement qui est toujours déjà sacrifice, Stourdzé relance la question de la technique et du temps. La relation entre usure et usage mobilise la temporalité contre la baisse du taux de profit: «la société industrielle fait jouer par la circulation des marchandises les rites de détérioration et régénération. C'est un des biais par lequel le problème de la désorganisation est occulté, déplacé, censuré» (Stourdzé, 1973, 87-88).

En dévoilant cette occultation, Stourdzé souligne une nouvelle fois qu'il n'y pas de fondement sans sacrifice originel (Stourdzé, 1973, 96), mais que le sacrifice produit toujours de la valeur. Tout dépend donc de son organisation qui mobilise deux formes : l'une interne, relative à la fondation du groupe, qui se fait aux dépens du travailleur et l'autre externe, relative à la circulation de l'échange, qui s'affirme dans l'investissement comme dépense de la réserve. La notion « d'inflation sacrificielle» (Stourdzé, 1973, 99) s'avère particulièrement importante pour envisager un passage du cycle de la valeur économique au monde des valeurs. L'inflation sacrificielle se mesure à l'augmentation du nombre des victimes et à l'intensité de l'austérité. Cette comptabilité mesure le sacrifice comme la soumission au règne de la quantité et inscription des signes sur les corps. Stourdzé replace l'histoire de l'industrialisation et plus globalement l'acte de produire comme résultat probable «d'un interdit porté sur la bestialité qui est significatif du désir de la production de l'organisation » (Stourdzé, 1973, 99). Cette position paraît audacieuse d'un point de vue anthropologique, en soulignant l'importance du désir dans la formation de la production.

«Une inflation monstrueuse et terrifiante s'empare de cette logique du sacrifice lorsqu'une organisation qui prétend au pouvoir y parvient. Incapable de se saisir dans un premier temps des rouages mécaniques de l'appareil, elle ne peut compter que sur la seule légitimité sacrificielle. La terreur met en place son dispositif. Le pouvoir du sacrifice cherche à contrebalancer les faiblesses de l'organisation. »(Stourdzé, 1973, 99).

L'inflation sacrificielle est l'expression d'une crise, toujours nécessaire avant le retour à l'équilibre. On comprend dès lors qu'aussi éloignés qu'ils paraissent, la guerre et le luxe sont des «stimulateurs du sacrifice» (Stourdzé, 1973,105). La guerre est une destruction massive comme la dépense ostentatoire de la classe loisir (Veblen, 1970) est un gaspillage sans limite. L'inflation sacrificielle se développe avec les combats et les crises de surproduction. Aussi, «L'histoire des quarante dernières années retrace les péripéties de cette inflation sacrificielle. Le délire du signe morbide renvoie en raccourci au délire de l'organisation. À la fin du processus le pouvoir tient en ses mains la mort de la collectivité entière. »(Stourdzé, 1973, 99).

Pour échapper à la mort la collectivité recourt, après la Seconde Guerre mondiale, à la dissuasion nucléaire et à limiter l'extension des conflits. Stourdzé entend poser, à l'aide de l'inflation sacrificielle, la question de la dépense et des valeurs dont il convient impérativement de faire la mesure. Il ne s'agit plus d'opposer la contemplation à l'activité, mais de calculer le coût de 
l'intégration ou de la désintégration des collectifs en dehors des rites primitifs et mythologiques de la destruction et de la renaissance.

\section{L'anti-organisation comme question non résolue}

Afin de rendre compte du dernier mouvement de la pensée de Stourdzé dans le livre et de montrer comment va évoluer l'appréciation de l'antiorganisation, partons d'une citation: «Si nous ne détruisons pas les organisations, elle nous briserons définitivement. Disloquer l'organisation, c'est briser les champs qu'elle innerve et nourrit, c'est inventer un temps et un espace autres, c'est dissoudre les formes de l'équivalence qui nous enserrent et nous emprisonnent, pulvériser le système de signes qui rend la vie monnayable sous forme de salaires et de marchandise, revendiquer un temps sans mode ni plein emploi. » (Stourdzé, 1973, 159).

Ce diagnostic impitoyable des organisations aliénantes révèle la nécessité de construire de nouveaux espaces et une temporalité neuve face à la « dégénérescence » qui s'explique à travers la relation entre le «corps et l'organisation » (Stourdzé, 1973, 109). C'est donc à la recherche de nouvelles valeurs que part Stourdzé, d'une alternative aux formes de l'équivalence générale basée sur la circulation des signes. Il va préciser ainsi son travail critique de déconstruction de l'organisation.

\section{Le contrôle des corps: du calcul à la maîtrise des énergies organisationnelles}

Contrôle et comptabilité deviennent des éléments clés de l'appréciation et de l'évaluation des corps dans l'organisation. Ces opérations reposent sur de nouveaux outils : tests, cartes et sociogrammes. Les ordinateurs seront mobilisés pour cette opération : «le rôle des ordinateurs devient central » car désormais "toutes opérations sont désormais traduites en langage machine » (Stourdzé, 1973,111). Comment expliquer que de nouveaux territoires apparaissent pourtant pour alors que le centre, dont nous avons vu qu'il était sacré, devient occupé par une machine?

Stourdzé recourt à Freud pour décrire le processus organisationnel énergétique, selon un mécanisme de décharge qui structure toute circulation. $\mathrm{La}$ relation entre énergie et circulation repose sur un double mouvement: la décharge contrôlée par « liaison » (où se réalise l'investissement) et la décharge agissant par report (où se réalise l'accumulation) à l'origine du surplus. Le report in fine apparaît comme la garantie de l'organisation, de son développement, de sa croissance. À l'origine de la formation d'un surplus, le report introduit un déséquilibre dont le contrôle n'est pas aisé; il est à l'origine des changements. Là résident des potentiels de rupture. C'est la gestion des « surplus » qui menace les fondements de l'organisation: «la plus-value vient représenter les décharges énergétiques qui tentent d'entrer avec effraction dans les dispositifs organisationnels. » (Stourdzé, 1973,113)

Dans son mouvement interne l'organisation, non seulement guide le passage de l'énergie vers le psychisme, mais intègre des poussées diverses dans des dispositifs collectifs. Ce phénomène renouvelle, par un emprunt à la psychanalyse, l'approche de la production et plus globalement 
de l'industrie comme nous allons l'observer.

\section{Innovation et énergie libre : le réglage d'un déséquilibre}

Une nouvelle étape de l'organisation est franchie avec l'éloignement des corporations de l'Ancien Régime: le nouveau qui était contraire aux règlements devient un élément clé. «La révolution industrielle n'a été rendue possible que par l'acceptation par les organisations des innovations » (Stourdzé, 1973, 115). Sur cette base, il est aisé de comprendre que le déséquilibre organisationnel devient un principe de la métastabilité, c'est-àdire d'une impermanence qui sera autoentretenue. La libération de la concurrence laisse se déployer une énergie dans une production que théorisera Schumpeter avec la « destruction créatrice». Le commerce absorbe la violence de la guerre sur laquelle reposait l'Ancien Régime. Cependant, si l'innovation et le déséquilibre de l'organisation dépendent de l'énergie libre, le besoin de rupture se combine à une récupération de cette énergie; le destin de l'énergie se trouve ainsi pris au piège et implique une pharmacologie pour soigner par des thérapies les maladies organisationnelles. En effet les dysfonctionnements impliquent, après la recherche des différences entre le pur et l'impur, l'épuration des zones différentielles. La bonne circulation des signes impose un retour à la restructuration de l'organisation, de telle sorte que les «zones obscures, les frontières sont liquidées » (Stourdzé, 1973,111) pour laisser la place à une communication fluide que régule l'ordinateur.

\section{La récupération et le refoulement du "libre"}

Le concept de récupération de l'énergie libre enraye le chemin vertueux de l'organisation et permet de revenir sur la question de l'émancipation limitée par le biais de l'énergie liée. Son traitement demeure décisif pour comprendre la fin de l'ouvrage. «L'organisation reprend à son compte les dépenses improductives; de fait il s'agit bien de contourner de l'énergie libre, d'en censurer les caractéristiques, d'escamoter le divorce entre énergie libre et énergie liée et de réinsérer cette énergie dans le dispositif organisationnel comme moteur. Le variable donne la vie au constant, l'instabilité doit nourrir l'équilibre. Le concept de récupération trouve ici sa place. L'organisation opère pour son compte la logique $d u$ refoulement dont rêvaient les réformateurs sociaux. » (Stourdzé, 1973, 121)

Le dispositif organisationnel entrelace ici les dimensions économique, psychique et politique. Le thème de l'anti-organisation ne s'entend pas alors comme une rupture; ce n'est plus le travail du négatif, au sens de Hegel et de Marx, qui se trouve revendiqué. Dès lors, relève Stourdzé, le thème de la participation comme accompagnement nécessaire à la circulation de la production devient un élément de l'idéologie qui renforce la coordination à travers le management. Stourdzé souligne ainsi que la participation consiste à «empêcher les membres d'élaborer d'autres perspectives que celles désirées par le centre » (Stourdzé, 1973, 116).

Cette position peut paraître en contradiction face à l'affirmation d'une possible liberté préalablement revendiquée. Stourdzé s'attache, en effet, dans les parties ultimes de l'ouvrage à souligner les limites de la contestation. La capture de l'énergie libre ou déliée annonce la fin du discours 
radical et anticapitaliste. Le désir de révolution échoue dans son « désir de blocage » des organisations. Si l'organisation s'avère vulnérable par les tensions qui s'y exercent, les tentatives de blocage demeurent inopérantes. Entre la grève sauvage et le mythe de la grève générale, la volonté d'une suspension de l'échange inégal paraît vain face aux régulations complexes du système qui ramène des normes, où les victoires de la contestation ne sont que transitoires. Les modèles des révolutions de 1789 ou 1917 sont convoqués pour confirmer que le centre reprend toujours le pouvoir en désamorçant l'esprit de révolte. Un point décisif est à relever dans ce constat, l'inefficacité d'un discours politique de type prophétique :

« La prophétie crée la tension, elle polarise le désir, le capte et l'organise. Il est remarquable de constater comment une prophétie comme celle du socialisme permet de "retarder" à l'infini les preuves de sa réalisation. [...] Le non-accomplissement de la prophétie renforce l'organisation car elle témoigne de la puissance des forces multiples qui se liguent pour empêcher la réalisation de la promesse. [...] L'organisation prophétique, comme organisation de l'attente, peut reproduire ainsi le spectacle d'une prophétie indéfiniment renouvelée et jamais réalisée. » (Stourdzé, 1973,147).

Si chaque situation de crise présente le profil d'une anti-organisation, la dislocation portée des expériences spontanées est toujours stoppée. La temporalité du prophétisme est toujours la promesse d'un advenir qui n'arrive pas. Le temps court de l'énergie libre redevient de l'énergie liée quand la révolte se trouve désamorcée. À cela il faut ajouter que le temps long du prophète apporte un espace de respiration. Sa parole sert alors à simuler dans l'intermittence des crises un esprit libre qui transitoirement régénère l'organisation : «La prophétie sert ainsi de moteur à l'organisation. L'énergie rebondit d'objectif intermédiaire en objectif intermédiaire pour le plus grand profit de l'organisation ». (Stourdzé, 1973, 148) La collectivité n'assume plus que les désirs que l'organisation détourne à son profit. Stourdzé tente alors d'établir une loi des crises : «plus le sacrifice est grand, plus proche est la réalisation de la prophétie » (Stourdzé, 1973,153). Ajoutons, en complément, que l'emprise du discours prophétique annonce une détérioration des conditions d'existence, sinon l'imminente d'une catastrophe.

\section{Conclusion : l'organisation comme tournant d'une pensée critique}

La crise est un moment nécessaire de l'organisation, c'est le moment de sa revitalisation. À l'action dissolvante de l'intérieur et à la contamination de l'extérieur, l'organisation ne peut qu'opposer une fermeture et un renforcement de transition pour se maintenir. Difficile de ne pas voir dans ce constat issu des théories de l'information une réflexion politique, après l'échec des événements de mai 68, auxquels Stourdzé s'était impliqué personnellement en tant que membre du mouvement du 22 mars. Le travail critique chez lui ne se limite pas à promouvoir une anti-organisation. L'originalité de sa démarche se précise dans une anthropologie, où le sacrifice tient une place centrale. La volonté d'un temps soustrait à la consommation lui permet de réexaminer la logique sacrificielle. Quelle limitation apporter 
aux crises et à l'inflation sacrificielle qu'elles organisent?

La crise traduit une désintégration, un désordre. Stourdzé note que l'argent accélère le «processus de dissolution » (Stourdzé, 1973, 150). L'argent en se transformant en capital, après la phase d'accumulation primitive, devient un instrument de désintégration et non plus seulement d'échange. Dès lors, le problème va être de penser de manière originale la transformation organisationnelle comme la synthèse de l'innovation de la prospective. Nous nous trouvons à un tournant de sa trajectoire dans les années 80 .

Sur le plan conjoncturel, la fin de l'espérance d'une suppression du capitalisme ne supprime pas toute motivation pour l'action chez Stourdzé. Les conclusions du livre, au contraire, préparent l'implication dans le CESTA (Centre d'Études des Systèmes et Technologies Avancées) de 1979 à 1986, dont il sera le directeur. Sur le plan théorique, c'est bien le deuil d'une théorie révolutionnaire issue d'un marxisme hétérodoxe qui s'exprime. Pour autant la recherche d'une alternative continuera, elle prendra d'autres formes à travers une critique des élites et la construction d'un imaginaire (Stourdzé, 1987). Une politique symbolique s'avère chez lui indispensable à l'investissement psychique et à la dynamique des groupes qui ont besoin d'image mobilisatrice (Sfez, 1978). Comment concilier tensions et apaisements dans une organisation qui cherche son propre rythme. La régulation des cycles ne peut s'élaborer que dans l'interstice, où les relations entre organisation et territoire deviennent importantes. C'est là, pensons-nous, que se développera progressivement la dimension géostratégique de la prospective que défendra Stourdzé en particulier autour du programme Eurêka, avant de disparaître prématurément. La construction d'une « zone différentielle » dans l'organisation sera toujours chez lui un objectif qui laisse un champ à la gratuité et à la coopération toujours présente au cœur de la violence des échanges. De façon originale et toujours actuelle, il conciliera le réalisme des programmes technologiques avec une ouverture des possibles dans sa façon d'aborder la prospective. C'est son anthropologie du sacrifice qui dévoile le besoin d'une économie élargie, où l'incalculable est un moment nécessaire de l'échange et où la logique du don n'est pas effacée par le prix. Les controverses contemporaines sur le statut des communs sont préfigurées dans une telle orientation.

L'enseignement de Stourdzé anticipe les questions majeures de notre période de transition comme le passage de la mécanique à l'informatique, ou encore le passage de la diffusion à la commutation. Les NBIC (Nanotechnologies, biotechnologies, informatique et sciences cognitives) créent une rupture, où la connaissance du principe de récupération alliée à la loi de l'inflation sacrificielle autorise la conception d'un chemin vertueux pour enrayer une désintégration qui serait maintenant fatale. Faisons ce pari, pour ne pas avoir le sentiment de la répétition d'une " étrange défaite », texte de Marc Bloch que Stourdzé appréciait particulièrement. 


\section{$R \cdot E \cdot F \cdot E \cdot R \cdot E \cdot N \cdot C \cdot E \cdot S \quad R \cdot E \cdot S \cdot U \cdot M \cdot E ́$}

BATAILLE Georges (1949), La part maudite, Paris, éd. de Minuit.

BAUDRILLARD Jean (1980), De la séduction, Paris, Éditions Galilée.

CLOUSCARD Michel (1981), Le Capitalisme de la séduction - Critique de la social-démocratie, Paris, Éditions sociales.

MARCH \& SIMON Herbert (1960), Les organisations, Paris, Dunod.

MAUSS Marcel (1968), Euvres, Tome 1, Les fonctions sociales du sacré, Paris, éd. de Minuit. NAVILLE Pierre (1963), Vers l'automatisme social, Paris, Gallimard.

SAUVY Alfred (1980), La machine et le chômage, Paris, éd. Dunod.

SFEZ Lucien (1973), Critique de la décision, Paris, Presses de la Fondation nationale des sciences politiques.

SFEZ Lucien (1978), L'enfer et le paradis, Paris, Puf.

SIK Ota (1974), La troisième voie, traduction française, Paris, Gallimard.

STOURDZÉ Yves (1973), Organisation, anti-organisation, Tours, Maison Mame, (2015) Sens\&Tonka.

STOURDZÉ Yves (1986), Pour une poignée d'électrons, Pouvoir et communication, Paris, Fayard, (2016) Sens\&Tonka.

VEBLEN Thorstein (1970), Théorie de la classe loisir (1899), Paris, Gallimard.
Partant d'une relecture d'un ouvrage (Stourdzé, 1973), nous soulignerons à la fois l'apport des propositions contenues dans cet opus et la richesse des perspectives qu'il ouvre. Dans la première partie de cet article, l'organisation apparait comme la question qui introduit une approche anthropologique originale du pouvoir. Dans la seconde partie nous verrons comment la mutation du système de production s'envisage comme une limitation du sacrifice. En conclusion, nous observerons en quoi la position de Stourdzé oscille entre une critique active et un pessimisme foncier. Cette ambivalence prépare une rupture qu'inscrira par la suite le CESTA. Stourdzé entrera finalement à la fin des années 70 dans une nouvelle phase de son parcours : il devient lui-même organisateur et pratiquera l'innovation et la prospective.

\footnotetext{
Abstract

Based on a rereading of a book by Stourdzé (1973), we will discuss the contribution it makes and the wealth of perspectives it opens up. In the first part of the article, the topic of the organization introduces an original anthropological approach to power. In the second part, we see how changes in the system of production can be understood as the limitation of sacrifice. Finally, we note how Stourdzé's position oscillates between an active critique and a fundamental pessimism. This ambivalence explains a rupture introduced by CESTA later. In the late 1970s, Stourdzé entered a new stage in his own intellectual trajectory, becoming an organizer and practitioner of innovation and forecasting.
} 